\title{
ON KERNELS OF FALTUNG TRANSFORMATIONS
}

\author{
BY \\ RALPH PALMER AGNEW
}

1. Introduction. A complex-valued function $J(t)$ defined over $-\infty<t<\infty$ being given, the function

$$
y(s)=\int_{-\infty}^{\infty} J(t) x(s+t) d t
$$

is, if it exists, called the faltung of the kernel $J(t)$ and the function $x(t)$. We use Lebesgue measure and integration, and let $L$ denote the class of complexvalued functions $x(t)$ integrable (and hence also absolutely integrable) over the infinite interval $-\infty<t<\infty$.

It is well known that if $J \varepsilon L$, then the faltung $y(s)$ of each $x \varepsilon L$ exists (that is, is finite) for almost all $s$, and $y \varepsilon L$. This is implied by the computation

$$
\begin{aligned}
\int_{-\infty}^{\infty} y(s) d s & =\int_{-\infty}^{\infty} d s \int_{-\infty}^{\infty} J(t) x(s+t) d t=\int_{-\infty}^{\infty} J(t) d t \int_{-\infty}^{\infty} x(s+t) d s \\
& =\left[\int_{-\infty}^{\infty} J(t) d t\right]\left[\int_{-\infty}^{\infty} x(s) d s\right],
\end{aligned}
$$

which is justified by the absolute convergence of the integrals involved. If $J(t)$ is an essentially bounded measurable function, say $|J(t)| \leqq M$ for almost all $t$, and $x \varepsilon L$, then the simple estimate

$$
\begin{aligned}
|y(s)| & \leqq \int_{-\infty}^{\infty}|J(t)||x(s+t)| d t \leqq M \int_{-\infty}^{\infty}|x(s+t)| d t \\
& =M \int_{-\infty}^{\infty}|x(t)| d t
\end{aligned}
$$

shows that $y(s)$ exists and is bounded over $-\infty<s<\infty$. Each of these results is of the type: If $J$ has property $P$, then $y$ has property $Q$ for each $x \varepsilon L$. To supplement such results, it is desirable to know whether the conclusion that $J$ has property $P$ can be drawn from the hypothesis that $y$ has property $Q$ whenever $x$ belongs to an appropriate class $X$ of functions. Doubtless the most pertinent questions are those for which the class $X$ is $L$ itself. We are able to obtain affirmative theorems not only when $X$ is the class $L$ but also when $X$ is a suitable class of step functions in $L$. Such theorems become stronger and

Presented to the Society, September 7, 1939; received by the editors July 18, 1939. 
throw more light on the real character of faltung transformations when the extent of the class $X$ is reduced. There is some arbitrariness in choice of the classes $X$; we endeavor to make them at the same time as simple and illuminating as possible.

An example may serve to illustrate a role played by step functions in the theory of faltung transformations. If $J(t)=\exp i t^{n}, n>2$, then (see $\S$ ) simple estimates show that the faltung of each ordinary step function is a bounded continuous function in class $L$. But Theorem 3.1 shows that there exist generalized step functions in class $L$ of which the faltung is not in class $L$.

The main results of this paper are Theorems $2.1,3.1$, and 4.1 which are of the following type: If $y(s)$ has property $P$ for each $x(t)$ belonging to a class $X$ of functions, then $J(t)$ must have property $Q$. With each of these theorems is associated a theorem of familiar type which asserts that, if $J$ has property $Q$, then (i) $y$ has property $P$ for each $x \varepsilon L$ and (ii) a certain constant determined by $J$ is the bound of the transformation, that is, the least constant $M$ such that a constant (norm) determined by $y$ is less than or equal to $M \int_{-\infty}^{\infty}|x(t)| d t$ for each $x \varepsilon L$.

The class $X$ is in each case a nonlinear subclass of $L$ consisting of certain generalized non-negative step functions. Neither the class $X$, nor the larger manifold $\mathfrak{M}(X)$ consisting of all finite linear combinations of elements of $X$, forms a closed set in the space $L$ in which the distance between two elements $x_{1}(t)$ and $x_{2}(t)$ of $L$ is given by the familiar metric

$$
\int_{-\infty}^{\infty}\left|x_{2}(t)-x_{1}(t)\right| d t
$$

in other words the space obtained by using the elements of $\mathfrak{M}(X)$ and the metric of $L$ is not complete. It is shown in $\$ 6$ that each of Theorems $2.1,3.1$, and 4.1 will fail if $X$ is replaced by certain smaller classes of step functions.

Let $S$ denote the special class of all real non-negative functions $x=x(\bar{t})$ such that (i) $x \varepsilon L$ and (ii) there exist non-negative constants $\cdots, c_{-1}, c_{0}, c_{1}, c_{2}, \cdots$ and $\cdots<a_{-1}<a_{0}<a_{1}<a_{2}<\cdots$ (depending on the particular function $x$ ) such that $\lim _{n \rightarrow-\infty} a_{n}=-\infty, \lim _{n \rightarrow \infty} a_{n}=\infty$, and for each $n=\cdots,-1,0,1$, $2, \cdots$,

$$
x(t)=c_{n}, \quad a_{n} \leqq t<a_{n+1} .
$$

Each $x \varepsilon S$ may be described as a real non-negative function in $L$ which is a generalized step function $\left({ }^{(1)}\right.$ having a finite number of steps in each finite interval.

Let $S_{U}$ denote the subclass of $S$ consisting of those functions in $S$ for which $a_{n+1}-a_{n}=1, n=0, \pm 1, \pm 2, \cdots$; each $x \varepsilon S_{U}$ is a unit step function, each step

(1) We reserve the term ordinary step function for step functions which vanish outside some finite interval. 
being one unit long. Each $x \varepsilon S$ is bounded over each finite interval, and each $x \varepsilon S_{U}$ is bounded over $-\infty<t<\infty$. (It is a trivial remark that the last assertion would be false if in (1.5) $a_{n} \leqq t<a_{n+1}$ were replaced by $a_{n}<t<a_{n+1}$.)

2. Conditions for existence of $y(s)$. This section is devoted to discussion and proof of the following two theorems.

Theorem 2.1. If $J(t)$ is such that, for each $x \in S_{U}$,

$$
y(s)=\int_{-\infty}^{\infty} J(t) x(s+t) d t
$$

exists for at least one $s$ in the interval $-\infty<s<\infty$, then $J(t)$ is measurable $\left({ }^{2}\right)$ over $-\infty<t<\infty$ and for each constant $0<A<\infty$ there is a constant $M_{A}$ such that

$$
\underset{-\infty<u<\infty}{\text { l.u.b. }} \int_{u}^{u+A}|J(t)| d t=M_{A}<\infty .
$$

TheOREM 2.2. If $J(t)$ is measurable and (2.12) holds, then for each $x \varepsilon L$, $y(s)$ defined by (2.11) exists for almost all s and is measurable, and for each $A>0$

$$
\underset{-\infty<u<\infty}{\text { l.u.b. }} \int_{u}^{u+A}|y(s)| d s \leqq M_{A} \int_{-\infty}^{\infty}|x(t)| d t
$$

where $M_{A}$ is the constant of (2.12). Moreover the constant $M_{A}$ in (2.21) is the best possible one in the sense that if a measurable function $J(\bar{t})$ satisfying $(2.12)$ and $A>0$ are fixed, then, for each $C<M_{A}$,

$$
\underset{-\infty<u<\infty}{\text { l.u.b. }} \int_{u}^{u+A}|y(s)| d s>C \int_{-\infty}^{\infty}|x(t)| d t
$$

will be true for some $x \varepsilon L$.

If $A_{1}$ and $A_{2}$ are finite positive numbers, then each interval $u \leqq t \leqq u+A_{1}$ can be covered by a finite set of intervals of the form $u_{k} \leqq t \leqq u_{k}+A_{2}$; hence it is apparent that if the left member of (2.12) is finite for some one $A>0$, then it is finite for each $A>0$. Therefore the condition (2.12) is equivalent to

$$
\underset{-\infty<u<\infty}{\text { l.u.b. }} \int_{u}^{u+1}|J(t)| d t<\infty,
$$

and this condition is easily seen to be equivalent to

$$
\underset{n=0, \pm 1, \pm 2, \cdots}{\text { l.u.b. }} \int_{n}^{n+1}|J(t)| d t<\infty .
$$

(2) Perhaps little would be lost if we were to assume measurability of $J(t)$; but the proof of measurability of $J(t)$ is so simple (see the few lines following the statement of Lemma 2.3) that we elect to prove it rather than to assume it. 
In $\S 7$ we discuss further the class of functions satisfying the inequality (2.12).

It is a corollary of Theorems 2.1 and 2.2 that if $J$ is such that $y(s)$ exists for at least one $s$ whenever $x \varepsilon L$, then $y(s)$ must exist for almost all $s$ whenever $x \varepsilon L$. This does not imply that if $J(t)$ and $x(t)$ are a pair of functions with $x \varepsilon L$ for which $y(s)$ exists for at least one $s$, then $y(s)$ must exist for almost all $s$; indeed if $J(t)$ is 0 or $t^{2}$ according as $[t]$, the greatest integer less than or equal to $t$, is even or odd, and $x(t)$ is $1 /\left(1+t^{2}\right)$ or 0 according as $[t]$ is even or odd, then $x \varepsilon L$ and $y(s)=0$ when $s$ is an integer but $y(s)=\infty$ when $s$ is not an integer.

Our first step in the proof of Theorem 2.1 is to prove

LEMмA 2.3. If $J(t)$ is such that, for each $x \varepsilon S_{U}, y(s)$ exists for at least one $s$, then $J(t)$ is integrable over each finite interval $a \leqq t \leqq b$ and, for each $x \varepsilon S_{U}, y(s)$ exists for at least one $s$ in each closed interval of unit length in the interval $-\infty<s<\infty$.

To prove Lemma 2.3, let $J(t)$ satisfy its hypothesis and let $x_{0}(t)$ be a positive function $x \varepsilon S_{U}$, say $x_{0}(t)=1 /\left(1+[t]^{2}\right)$. Let $s_{0}$ be fixed such that

$$
y_{0}\left(s_{0}\right)=\int_{-\infty}^{\infty} J(t) x_{0}\left(s_{0}+t\right) d t
$$

exists. Then if $-\infty<a<b<\infty$,

$$
\int_{a}^{b} J(t) x_{0}\left(s_{0}+t\right) d t
$$

exists. But the function $1 / x_{0}\left(s_{0}+t\right)$ is measurable and bounded over $a \leqq t \leqq b$; therefore $J(t)=\left[J(t) x_{0}\left(s_{0}+t\right)\right] / x_{0}\left(s_{0}+t\right)$ is integrable as well as measurable over $a<t<b$.

Now let an arbitrary function $x \varepsilon S_{U}$ be fixed. The function $X(t)$ defined by the series

$$
X(t)=\sum_{n=-\infty}^{\infty} 2^{-|n|} x(t+n)
$$

exists for almost all $t$, and $X \varepsilon S_{U}$; that $X \varepsilon L$ is shown by the computation

$$
\begin{aligned}
\int_{-\infty}^{\infty} X(t) d t & =\int_{-\infty}^{\infty} \sum_{n=-\infty}^{\infty} 2^{-|n|} x(t+n) d t \\
& =\sum_{n=-\infty}^{\infty} 2^{-|n|} \int_{-\infty}^{\infty} x(t+n) d t=3 \int_{-\infty}^{\infty} x(t) d t
\end{aligned}
$$

which is justified by the fact that $x(t) \geqq 0$ and $x \varepsilon L$; and $X(t)$ and $x(t)$ are constant over the same unit intervals. Let $s_{0}$ be fixed such that 


$$
Y\left(s_{0}\right)=\int_{-\infty}^{\infty} J(t) X\left(s_{0}+t\right) d t
$$

exists. Then

$$
\int_{-\infty}^{\infty} \sum_{n=-\infty}^{\infty} 2^{-|n|}|J(t)| x\left(s_{0}+n+t\right) d t
$$

exists, and since each term in the sum is measurable and non-negative, this implies that

$$
\int_{-\infty}^{\infty}|J(t)| x\left(s_{0}+n+t\right) d t
$$

exists for each $n$. Thus

$$
y(s)=\int_{-\infty}^{\infty} J(t) x(s+t) d t
$$

exists when $s=s_{0}, s_{0} \pm 1, s_{0} \pm 2, \cdots$. Since each closed unit interval contains at least one of these points, Lemma 2.3 is proved.

To complete the proof of Theorem 2.1 , let $J(t)$ satisfy the hypothesis of Theorem 2.1 and hence the conclusion of Lemma 2.3. To establish (2.12), we assume that (2.12) fails and obtain a contradiction. Failure of (2.12) implies that the left member of $(2.24)$ is $+\infty$; hence there is a sequence $n_{1}, n_{2}, n_{3}, \ldots$ of integers such that $\left|n_{p}-n_{q}\right|>3, p \neq q$, and

$$
\lim _{\alpha \rightarrow \infty} I\left(n_{\alpha}\right)=\infty
$$

where

$$
I(n)=\int_{n}^{n+1}|J(t)| d t
$$

It follows from (2.37) that we can choose a decreasing sequence $\theta_{1}>\theta_{2}>\ldots$ of positive numbers such that

$$
\sum_{\alpha=1}^{\infty} I\left(n_{\alpha}\right) \theta_{\alpha}=\infty, \quad \sum_{\alpha=1}^{\infty} \theta_{\alpha}<\infty .
$$

Let

$$
\begin{aligned}
x(t) & =\theta_{\alpha}, & & n_{\alpha}-1 \leqq t<n_{\alpha}+2, \alpha=1,2, \cdots, \\
& =0, & & \text { otherwise. }
\end{aligned}
$$

Then $x(t)$ is real, non-negative, and constant over each of the abutting unit intervals $n \leqq t<n+1$; and the second of the relations (2.38) implies that $x \varepsilon L$. 
Hence $x \varepsilon S_{U}$. Since the integrands are all measurable and non-negative, we find when $|s| \leqq 1$

$$
\begin{aligned}
\int_{-\infty}^{\infty}|J(t)||x(s+t)| d t & =\int_{-\infty}^{\infty}|J(t-s)||x(t)| d t \\
& =\sum_{\alpha=1}^{\infty} \int_{3 n_{\alpha}-1}^{n_{\alpha}+2}|J(t-s)||x(t)| d t \\
& =\sum_{\alpha=1}^{\infty} \theta_{\alpha} \int_{3 n_{\alpha}-1}^{n_{\alpha}+2}|J(t-s)| d t \\
& \geqq \sum_{\alpha=1}^{\infty} \theta_{\alpha} \int_{n_{\alpha}}^{n_{\alpha}+1}|J(t)| d t=\sum_{\alpha=1}^{\infty} \theta_{\alpha} I\left(n_{\alpha}\right)=\infty ;
\end{aligned}
$$

hence

$$
y(s)=\int_{-\infty}^{\infty} J(t) x(s+t) d t
$$

fails to exist when $|s| \leqq 1$ and we have a contradiction of the fact that $y(s)$ must exist for at least one $s$ in each unit interval. This completes the proof of Theorem 2.1.

Proof of the first part of Theorem 2.2 is very simple. Assuming that $J(t)$ is measurable and (2.12) holds, and that $x \varepsilon L$ and $A>0$ are fixed, we find for each real $u$

$$
\begin{aligned}
\int_{u}^{u+A}|y(s)| d s & \leqq \int_{u}^{u+A} d s \int_{-\infty}^{\infty}|J(t) x(s+t)| d t \\
& =\int_{u}^{u+A} d s \int_{-\infty}^{\infty}|J(t-s)||x(t)| d t \\
& =\int_{-\infty}^{\infty}|x(t)| d t \int_{u}^{u+A}|J(t-s)| d s \\
& =\int_{-\infty}^{\infty}|x(t)| d t \int_{t-u-A}^{t-u}|J(\alpha)| d \alpha \leqq M_{A} \int_{-\infty}^{\infty}|x(t)| d t
\end{aligned}
$$

the steps are easily justified by fundamental theorems which imply also that $y(s)$ exists almost everywhere and is measurable over $u \leqq s \leqq u+A$. It follows immediately that $y(s)$ exists almost everywhere and is measurable over $-\infty<s<\infty$, and that (2.21) holds.

In our proof of the last part of Theorem 2.2 we shall use the following lemma in which we choose notation to fit the application.

LemMA 2.4. If $u$ is real, $A>0, h>0$, and $J(t)$ is integrable over the interval $u \leqq t \leqq u+A+h$, then 


$$
\lim _{\delta \rightarrow 0+} \int_{u}^{u+A} d s \frac{1}{\delta} \int_{s}^{s+\delta}|J(t)-J(s)| d t=0 .
$$

In case $J(t)$ is continuous over $u \leqq t \leqq u+A+h$ we can, for each $\epsilon>0$, choose $\delta_{0}>0$ such that $|J(t)-J(s)|<\epsilon / A$ when $s$ and $t$ lie between $u$ and $u+A+\delta_{0}$ and $|t-s|<\delta_{0}$; letting $I(\delta)$ denote the iterated integral in (2.41), we find that $0 \leqq I(\delta)<\epsilon$ when $0<\delta<\delta_{0}$ and (2.41) follows. In case $J(t)$ is not continuous, we can show that lim $\sup _{\delta \rightarrow 0} I(\delta)<\epsilon$ by use of the following inequality:

$$
|J(t)-J(s)| \leqq\left|J(t)-J_{\epsilon}(t)\right|+\left|J_{\epsilon}(t)-J_{\epsilon}(s)\right|+\left|J_{\epsilon}(s)-J(s)\right|
$$

in which $J_{\epsilon}(t)$ is a function continuous over $u \leqq t \leqq u+A+h$ for which

$$
\int_{u}^{u+A+h}\left|J(t)-J_{\epsilon}(t)\right| d t<\epsilon / 3 .
$$

Let a measurable function $J(t)$ for which (2.12) holds and a constant $A>0$ be fixed. For each $\delta>0$, let $x_{\delta}(t)$ be defined by

$$
\begin{aligned}
x_{\delta}(t) & =\delta^{-1}, & & 0 \leqq t<\delta, \\
& =0, & & \text { otherwise. }
\end{aligned}
$$

Then

$$
\int_{-\infty}^{\infty}\left|x_{\delta}(t)\right| d t=1
$$

The faltung $y_{\delta}(s)$ of $J$ and $x_{\delta}$ is

$$
y_{\delta}(s)=\int_{-\infty}^{\infty} J(t) x_{\delta}(s+t) d t=\frac{1}{\delta} \int_{-8}^{-8+\delta} J(t) d t
$$

hence

$$
y_{\delta}(-s)-J(s)=\frac{1}{\delta} \int_{\delta}^{s+\delta}[J(t)-J(s)] d t
$$

so that

$$
\left|y_{8}(-s)-J(s)\right| \leqq \frac{1}{\delta} \int_{0}^{s+\delta}|J(t)-J(s)| d t
$$

and for each $u$

$$
\int_{u}^{u+A}\left|y_{\delta}(-s)-J(s)\right| d s \leqq \int_{u}^{u+A} d s \frac{1}{\delta} \int_{s}^{s+\delta}|J(t)-J(s)| d t .
$$

Using Lemma 2.3, we obtain 


$$
\lim _{\delta \rightarrow 0} \int_{u}^{u+A}\left|y_{\delta}(-s)-J(s)\right| d s=0
$$

and this implies that

$$
\lim _{\delta \rightarrow 0} \int_{u}^{u+A}\left|y_{\delta}(-s)\right| d s=\int_{u}^{u+A}|J(s)| d s .
$$

If now $C<M_{A}$, then we can choose a fixed $u$ such that

$$
\int_{u}^{u+A}|J(s)| d s>C
$$

and then because of (2.44) and (2.45) we can choose a fixed $\delta>0$ such that

$$
\int_{u}^{u+A}\left|y_{\delta}(-s)\right| d s>C .
$$

Using (2.42), we can write $(2.46)$ in the form

$$
\int_{-u-A}^{-u}\left|y_{\delta}(s)\right| d s>C \int_{-\infty}^{\infty}\left|x_{\delta}(t)\right| d t
$$

this implies (2.22) and Theorem 2.2 is proved.

The hypotheses of Theorems 2.1 and 2.2 do not imply that, if $x \varepsilon L$, then $y(s)$ must exist for all real $s$. This follows from

TheOREM 2.5. In order that $J(t)$ may be such that

$$
y(s)=\int_{-\infty}^{\infty} J(t) x(s+t) d t
$$

exists for all real $s$ whenever $x \varepsilon L$, it is necessary and sufficient that $J(t)$ be measurable and essentially bounded.

A function $J(t)$ is called essentially bounded if there is a constant $M$ such that $|J(t)|<M$ for almost all $t$. Sufficiency is a consequence of the well known fact that if $J(t)$ is measurable and essentially bounded and $\xi(t) \varepsilon L$, then $J(t) \xi(t) \varepsilon L$; and necessity is a consequence of the well known fact that if $J(t) \xi(t) \varepsilon L$ for each $\xi \varepsilon L$, then $J(t)$ is measurable and essentially bounded. If $J(t)$ is essentially bounded, then (2.12) holds and $M_{A} \leqq A \beta$ where $\beta$ is the least constant such that $|J(t)| \leqq \beta$ for almost all $t$; but (2.12) does not imply that $J(t)$ is essentially bounded.

3. Conditions for $y \varepsilon L$. It is possible to prove, by means of an extension of a theorem of Banach $\left.{ }^{3}\right)$ and some ideas which we use in the course of proof

$\left(^{8}\right)$ Banach, Theorie des Operations Linéaires, Warsaw, 1932, p. 87, Theorem 9. The extension required is from the finite interval $0 \leqq t \leqq 1$ to the infinite interval $-\infty<t<\infty$, and from realvalued functions to complex-valued functions. 
of Theorem 3.1, that if $J(t)$ is such that, for each $x \varepsilon L, y(s)$ exists for almost all $s$ and $y \varepsilon L$, then $J \varepsilon L$. Theorem 3.1 below, of which we give a direct proof, includes this result.

THEOREM 3.1. If $J(t)$ is such that, for each $x \varepsilon S$,

$$
y(s)=\int_{-\infty}^{\infty} J(t) x(s+t) d t
$$

exists for almost all $s$ and $y \varepsilon L$, then $J \varepsilon L$.

Theorem 3.2. If $J \varepsilon L$, then for each $x \varepsilon L, y(s)$ as defined by (3.11) exists for almost all s, $y \varepsilon L$, and

$$
\int_{-\infty}^{\infty}|y(s)| d s \leqq M_{\infty} \int_{-\infty}^{\infty}|x(t)| d t
$$

where

$$
M_{\infty}=\int_{-\infty}^{\infty}|J(t)| d t
$$

moreover $M_{\infty}$ is the best possible constant in (3.21) in the sense that if $C<M_{\infty}$ then

$$
\int_{-\infty}^{\infty}|y(s)| d s>C \int_{-\infty}^{\infty}|x(t)| d t
$$

will hold for some $x \varepsilon L$.

Our first step in the proof of Theorem 3.1 is to prove

LEMma 3.3. If $J(t)$ is such that $y \varepsilon L$ whenever $x \in S$, then there is a constant $M<\infty$ such that

$$
\int_{-\infty}^{\infty}|y(s)| d s \leqq M \int_{-\infty}^{\infty}|x(t)| d t, \quad x \varepsilon S_{1}
$$

where $S_{1}$ is the subclass of $S$ consisting of those functions $x(t)$ in $S$ which vanish outside the interval $0 \leqq t<1$.

If $J(t)$ satisfies the hypothesis of Lemma 3.3, and no $M<\infty$ exists for which (3.31) holds, then for each $n=1,2, \ldots$ there is $x_{n} \varepsilon S_{1}$ such that

$$
\int_{-\infty}^{\infty}\left|y_{n}(s)\right| d s>2^{n} \int_{-\infty}^{\infty}\left|x_{n}(t)\right| d t
$$

$y_{n}$ being the transform of $x_{n}$. Since the faltung transformation is homogeneous, we can assume that the functions $x_{n}(t)$ and $y_{n}(t)$ are divided by the left member of (3.32) so that 


$$
\int_{-\infty}^{\infty}\left|x_{n}(t)\right| d t<2^{-n}, \quad \int_{-\infty}^{\infty}\left|y_{n}(s)\right| d s=1
$$

Let $\lambda_{1}=0$ and choose constants $a_{2}>a_{1}+1$ such that the inequality

$$
\int_{a_{n}}^{a_{n+1}}\left|y_{n}\left(s-\lambda_{n}\right)\right| d s>1-3^{-n}
$$

holds when $n=1$. Since

$$
\lim _{\lambda_{n+1} \rightarrow \infty} \int_{a_{n+1}}^{\infty}\left|y_{n+1}\left(s-\lambda_{n+1}\right)\right| d s=1,
$$

we can choose $\lambda_{2}>\lambda_{1}+1$ and then choose $a_{3}>a_{2}+1$ in such a way that (3.34) holds when $n=2$. Continuation by induction furnishes sequences $a_{1}<a_{2}<a_{3}<\cdots$ and $\lambda_{1}<\lambda_{2}<\lambda_{3}<\cdots$ such that $a_{n+1}>a_{n}+1, \lambda_{n+1}>\lambda_{n}+1$, and (3.34) holds for each $n=1,2, \cdots$. Since $x_{n}(t) \varepsilon S_{1}$, it follows that $x_{n}\left(t-\lambda_{n}\right)$ vanishes outside the interval $\lambda_{n} \leqq t \leqq \lambda_{n}+1$. Let

$$
X(t)=\sum_{n=1}^{\infty} x_{n}\left(t-\lambda_{n}\right) \text {. }
$$

The series converges for each $t$ since, for each $t, x_{n}\left(t-\lambda_{n}\right) \neq 0$ for at most one $n$. Properties of the sequences $x_{n}$ and $\lambda_{n}$ imply that $X \varepsilon S$. Hence, by hypothesis,

$$
Y(s)=\int_{-\infty}^{\infty} J(t) X(s+t) d t
$$

exists for almost all $s$, and $Y \varepsilon L$. Since $X(t)$ vanishes at all points $t$ not in one of the mutually exclusive intervals $\left(\lambda_{n}, \lambda_{n}+1\right)$, it follows from (3.37) that

$$
\begin{aligned}
Y(s) & =\sum_{n=1}^{\infty} \int_{\lambda_{n}}^{\lambda_{n}+1} J(t-s) X(t) d t \\
& =\sum_{n=1}^{\infty} \int_{\lambda_{n}}^{\lambda_{n}+1} J(t-s) x_{n}\left(t-\lambda_{n}\right) d t=\sum_{n=1}^{\infty} \int_{-\infty}^{\infty} J(t-s) x_{n}\left(t-\lambda_{n}\right) d t \\
& =\sum_{n=1}^{\infty} \int_{-\infty}^{\infty} J(t) x_{n}\left(s-\lambda_{n}+t\right) d t=\sum_{n=1}^{\infty} y_{n}\left(s-\lambda_{n}\right) .
\end{aligned}
$$

It follows from (3.33) and (3.34) that for each $n=1,2, \ldots$

$$
\begin{array}{rlrl}
\int_{a_{n}}^{a_{n+1}}\left|y_{k}\left(s-\lambda_{k}\right)\right| d s & >1-3^{-n}, & k & =n, \\
& <3^{-k}, & k \neq n .
\end{array}
$$

Hence the inequality 


$$
|Y(s)|=\left|\sum_{k=1}^{\infty} y_{k}\left(s-\lambda_{k}\right)\right| \geqq\left|y_{n}\left(s-\lambda_{n}\right)\right|-\sum_{k \neq n}\left|y_{k}\left(s-\lambda_{k}\right)\right|
$$

implies that

$$
\int_{a_{n}}^{a_{n+1}}|Y(s)| d s \geqq 1-3^{-n}-\sum_{k \neq n} 3^{-k}=\frac{1}{2}, \quad n=1,2, \cdots .
$$

This is inconsistent with the previous conclusion that $Y \varepsilon L$; hence $M<\infty$ exists for which (3.31) holds and Lemma 3.3 is proved.

To prove Theorem 3.1, let $J(t)$ satisfy its hypothesis, and let $x_{\delta}(t)$ be the function in (2.42) which is $\delta^{-1}$ over $0 \leqq t<\delta$ and is 0 otherwise. If $0<\delta<1$, then $x_{\delta} \varepsilon S_{1}$; hence Lemma 3.3 implies existence of a constant $D<\infty$ such that

$$
\int_{-\infty}^{\infty}\left|y_{\delta}(s)\right| d s \leqq D \int_{-\infty}^{\infty}\left|x_{\delta}(s)\right| d s=D, \quad 0<\delta<1 .
$$

Since, by Theorem 2.1, $J(t)$ is integrable over each finite interval, (2.44) must hold; replacing $A$ by $2 A$ and setting $u=-A$ in (2.44) gives

$$
\int_{-A}^{A}|J(t)| d t=\lim _{\delta \rightarrow 0} \int_{-A}^{A}\left|y_{\delta}(-s)\right| d s, \quad A>0 .
$$

From (3.43) and (3.44) we obtain

$$
\int_{-A}^{A}|J(t)| d t \leqq D, \quad A>0,
$$

and this implies that

$$
\int_{-\infty}^{\infty}|J(t)| d t \leqq D
$$

Thus $J \varepsilon L$ and Theorem 3.1 is proved.

The first part of Theorem 3.2 is well known, and we give its proof merely for completeness. If $J \varepsilon L$ and $x \varepsilon L$, the computation

$$
\begin{aligned}
\int_{-\infty}^{\infty}|y(s)| d s & \leqq \int_{-\infty}^{\infty} d s \int_{-\infty}^{\infty}|J(t)||x(s+t)| d t \\
& =\int_{-\infty}^{\infty}|J(t)| d t \int_{-\infty}^{\infty}|x(s+t)| d t \\
& =\left[\int_{-\infty}^{\infty}|J(t)| d t\right]\left[\int_{-\infty}^{\infty}|x(s)| d s\right]
\end{aligned}
$$

is easily justified and (3.21) follows. If it be assumed that 


$$
\int_{-\infty}^{\infty}|y(s)| d s \leqq C \int_{-\infty}^{\infty}|x(t)| d t
$$

where

$$
C<\int_{-\infty}^{\infty}|J(t)| d t
$$

then we can set $D=C$ in (3.43) to obtain $D=C$ in (3.46) and have a contradiction of (3.49). Therefore if $C<M_{\infty}$, then $x \varepsilon L$ exists for which (3.23) holds and Theorem 3.2 is proved.

4. Conditions for $y \varepsilon B$. The measurable upper bound of a real measurable function $\xi(t)$ defined over $-\infty<t<\infty$ is the least number $\beta$ such that $\xi(t) \leqq \beta$ for almost all $t$. We write $\beta=$ m.u.b. $\xi(t)$; and let $B$ denote the class of all complex-valued measurable functions $x(t)$ for which m.u.b. $|x(t)|<\infty$.

THEOREM 4.1. If $J(t)$ is such that, for each $x \varepsilon S$,

$$
y(s)=\int_{-\infty}^{\infty} J(t) x(s+t) d t
$$

exists for almost all $s$ and $y \varepsilon B$, then $J \varepsilon B$.

Theorem 4.2. If $J \varepsilon B$, then for each $x \varepsilon L, y(s)$ as defined by (4.11) exists for all s and

$$
\underset{-\infty<s<\infty}{\text { l.u.b. }}|y(s)| \leqq \beta \int_{-\infty}^{\infty}|x(t)| d t
$$

where

$$
\beta=\underset{-\infty<t<\infty}{\operatorname{m.u.b.}}|J(t)|
$$

moreover $\beta$ is the best possible constant in (4.21) in the sense that, if $C<\beta$, then

$$
\underset{-\infty<s<\infty}{\text { l.u.b. }}|y(s)|>C \int_{-\infty}^{\infty}|x(t)| d t
$$

will hold for some $x \in L$.

Our first step in the proof of Theorem 4.1 is to prove the following lemma in which $S$ and $S_{1}$ denote the classes of step functions previously defined in $\S 1$ and Lemma 3.3.

LEMMA 4.3. If $J(t)$ is such that $y \varepsilon B$ whenever $x \varepsilon S$, then there is a constant $M$ such that

$$
\underset{-\infty<s<\infty}{\operatorname{m.u.b}}|y(s)| \leqq M \int_{-\infty}^{\infty}|x(t)| d t, \quad x \varepsilon S_{1} .
$$


To prove Lemma 4.3 , let $J(t)$ satisfy its hypothesis and assume that (4.31) fails. Failure of (4.31) implies existence for each $n=1,2,3, \cdots$ of a function $x_{n} \varepsilon S_{1}$ having a transform $y_{n}$ such that

$$
\underset{-\infty<s<\infty}{\text { m.u.b. }}\left|y_{n}(s)\right|>4^{n} \int_{-\infty}^{\infty} \cdot\left|x_{n}(t)\right| d t \text {. }
$$

We can suppose that each $x_{n}$, and hence also $y_{n}$, has been multiplied by the appropriate constant to give

$$
\underset{-\infty<s<\infty}{\text { m.u.b. }}\left|y_{n}(s)\right|=2^{n} ; \quad \int_{-\infty}^{\infty}\left|x_{n}(t)\right| d t \leqq 2^{-n}, \quad n=1,2, \cdots .
$$

If $\theta_{1}, \theta_{2}, \cdots$ is a sequence of which each element is either 0 or 1 , then

$$
X(t) \equiv \sum_{n=1}^{\infty} \theta_{n} x_{n}(t-n) \varepsilon S .
$$

Hence under our hypothesis

$$
Y(s) \equiv \int_{-\infty}^{\infty} J(t) X(s+t) d t \varepsilon B
$$

Starting with (4.34), we obtain

$$
Y(s)=\sum_{n=1}^{\infty} \theta_{n} y_{n}(s-n) \varepsilon B .
$$

That the conclusions just obtained are contradictory, and hence that Lemma 4.3 is true, is a consequence of the following lemma in which we write $w_{n}(s)$ for $y_{n}(s-n)$.

LEMMA 4.4. If $w_{n}(s)$ is a sequence of measurable functions, defined over $-\infty<s<\infty$, such that

$$
\underset{-\infty<s<\infty}{\text { m.u.b. }}\left|\sum_{n=1}^{\infty} \theta_{n} w_{n}(s)\right|<\infty
$$

for each sequence $\theta_{n}$ of which each element is 0 or 1 , then there is a constant $Q<\infty$ such that

$$
\underset{-\infty<s<\infty}{\operatorname{m.u.b}}\left|w_{n}(s)\right| \leqq Q, \quad n=1,2,3, \cdots \text {. }
$$

If $p$ is a positive integer and we set $\theta_{n}=0$ or 1 according as $n \neq p$ or $n=p$, we see that a constant $Q_{p}<\infty$ exists such that

$$
\underset{-\infty<s<\infty}{\operatorname{m.u.b}}\left|w_{p}(s)\right|=Q_{p} \text {. }
$$

To prove (4.42) amounts to proving that the sequence $Q_{p}$ is bounded. As- 
sume to the contrary that

$$
\limsup _{p \rightarrow \infty} Q_{p}=\infty .
$$

Setting $\theta_{n}=1$ for each $n$ in (4.41) shows that the series $\sum_{n=1}^{\infty} w_{n}(s)$ converges for almost all $s$; hence $\lim _{n \rightarrow \infty} w_{n}(s)=0$ for almost all $s$. Therefore by a theorem of Egoroff $\left.{ }^{4}\right) w_{n}(s)$ converges to 0 essentially uniformly over each set of finite measure $|E|$; that is, corresponding to each $\delta>0$, there is a subset $F$ of $E$ such that $|E-F|<\delta$ and $w_{n}(s)$ converges to 0 uniformly over $F$. Using (4.44), choose an index $n_{1}$ such that $R_{1} \equiv$ m.u.b. $\left|w_{n}(s)\right|>2$ when $n=n_{1}$. Let $E_{1}$ be a bounded set of positive measure such that $\left|w_{n}(s)\right|>1$ when $n=n_{1}$, $s \varepsilon E_{1}$. Let $F_{1}$ be a subset of $E_{1}$ such that $\left|E_{1}-F_{1}\right|<\left|E_{1}\right| / 2^{2}$ and $w_{n}(s)$ converges to 0 uniformly over $F_{1}$. Choose $n_{2}>n_{1}$ such that $\left|w_{n}(s)\right|<2^{-2}$ when $n=n_{2}, s \varepsilon F_{1}$ and also $R_{2} \equiv$ m.u.b. $\left|w_{n}(s)\right|>3+R_{1}$ when $n=n_{2}$. Let $E_{2}$ be a bounded set of positive measure such that $\left|w_{n}(s)\right|>2+R_{1}$ when $n=n_{2}, s \varepsilon E_{2}$. Let $F_{2}$ be a subset of $E_{1}+E_{2}$ such that $\left|E_{1}-F_{2}\right|<\left|E_{1}\right| / 2^{3},\left|E_{2}-F_{2}\right|<\left|E_{2}\right| / 2^{3}$, and such that $w_{n}(s)$ converges to 0 uniformly over $F_{2}$. Choose $n_{3}>n_{2}$ such that $\left|w_{n}(s)\right|<2^{-3}$ when $n=n_{3}, s \varepsilon F_{2}$, and also $R_{3} \equiv$ m.u.b. $\left|w_{n}(s)\right|>4+R_{1}+R_{2}$ when $n=n_{3}$. We continue by induction to obtain sequences of numbers and sets such that for each $p=1,2,3, \cdots$

$$
\begin{array}{cc}
\left|E_{p}\right|>0 ; \quad \underset{\substack{\text { m.u.b. } \\
-\infty<\infty<}}{ }\left|w_{n p}(s)\right|=R_{p} ; & \\
\left|w_{n_{p}}(s)\right|>p+\sum_{k=1}^{p-1} R_{k}, & s \varepsilon E_{p} ; \\
F_{p} \subset \sum_{k=1}^{p} E_{p} ; \quad\left|w_{n_{p}}(s)\right|<2^{-p}, & s \varepsilon F_{p-1} ; \\
\left|E_{k}-F_{p}\right| \leqq\left|E_{k}\right| / 2^{p+1}, & k \leqq p .
\end{array}
$$

Setting, for each positive integer $k, G_{k}=E_{k} F_{k} F_{k+1} F_{k+2} \cdots$, we find

so that by $(4.48)$

$$
E_{k}-G_{k}=E_{k}-\prod_{p=k}^{\infty} F_{p}=\sum_{p=k}^{\infty}\left(E_{k}-F_{p}\right)
$$

$$
\left|E_{k}-G_{k}\right| \leqq \sum_{p=k}^{\infty}\left|E_{k}-F_{p}\right| \leqq \sum_{p=k}^{\infty}\left|E_{k}\right| / 2^{p+1} \leqq\left|E_{k}\right| / 2
$$

and therefore $\left|G_{k}\right| \geqq\left|E_{k}\right| / 2>0$. Let

$$
W(s)=\sum_{p=1}^{\infty} w_{n_{p}}(s) .
$$

(4) See, for example, E. W. Hobson, The Theory of Functions of a Real Variable, vol. 2, p. 144. The extension of the theorem to complex-valued functions is easily made. 
For almost all $s$ in $G_{k}$ we find on using (4.45), (4.46), and (4.47) that

$$
\begin{aligned}
|W(s)| & \geqq-\sum_{p=1}^{k-1}\left|w_{n_{p}}(s)\right|+\left|w_{n_{k}}(s)\right|-\sum_{p=k+1}^{\infty}\left|w_{n_{p}}(s)\right| \\
& \geqq-\sum_{p=1}^{k-1} R_{p}+k+\sum_{p=1}^{k-1}\left|R_{p}\right|-\sum_{p=k+1}^{\infty} 2^{-p} \geqq k-1 .
\end{aligned}
$$

Hence for each integer $k$ there is a set of measure $\left|G_{k}\right|>0$ such that $|W(s)|$ $\geqq k-1$ for all $s$ in the set. Therefore

$$
\underset{-\infty<s<\infty}{\operatorname{m.u.b} .}\left|\sum_{p=1}^{\infty} w_{n_{p}}(s)\right|=\infty \text {. }
$$

But (4.49) contradicts the hypothesis that (4.41) must hold in case $\theta_{n}$ is 1 when $n=n_{1}, n_{2}, n_{3}, \cdots$ and 0 otherwise. This completes the proof of Lemma 4.4 and hence also that of Lemma 4.3.

To prove Theorem 4.1 , let $J(t)$ satisfy its hypothesis. By Lemma 4.3 there is a constant $D<\infty$ such that

$$
\underset{-\infty<s<\infty}{\operatorname{m.u.b}}\left|\int_{-\infty}^{\infty} J(t) x(s+t) d t\right| \leqq D \int_{-\infty}^{\infty}|x(t)| d t, \quad x \varepsilon S_{1} .
$$

Let, where $0<\delta<1, x_{\delta}(t)=\delta^{-1}$ when $0 \leqq t \leqq \delta$ and $x_{\delta}(t)=0$ otherwise. Then $x_{\delta} \varepsilon S_{1}$ and it follows from (4.51) that

$$
\underset{-\infty<s<\infty}{\text { m.u.b. }}\left|\frac{1}{\delta} \int_{\delta}^{s+\delta} J(t) d t\right|=\underset{-\infty<s<\infty}{\text { m.u.b. }}\left|\frac{1}{\delta} \int_{-s}^{-8+\delta} J(t) d t\right| \leqq D .
$$

But since $J(t)$ is integrable over each finite interval, $(1 / \delta) \int_{s}^{s+\delta} J(t) d t$ is a continuous function of $s$ for each $\delta>0$. Hence it follows from (4.52) that

$$
\left|\frac{1}{\delta} \int_{s}^{8+\delta} J(t) d t\right| \leqq D, 0<\delta<1,-\infty<s<\infty .
$$

But, by one form of the fundamental theorem of the calculus,

$$
\lim _{\delta \rightarrow 0}-\frac{1}{\delta} \int_{s}^{s+\delta} J(t) d t=J(s)
$$

for almost all $s$. Hence $|J(s)| \leqq D$ for almost all $s$ so that

$$
\beta=\underset{-\infty<t<\infty}{\text { m.u.b. }}|J(t)| \leqq D .
$$

Therefore $J \varepsilon B$ and Theorem 4.1 is proved.

To prove Theorem 4.2 , let $J \varepsilon B$ so that $\beta=$ m.u.b. $|J(t)|<\infty$. If $x \varepsilon L$, then for each $s$ 


$$
\begin{aligned}
|y(s)| & =\left|\int_{-\infty}^{\infty} J(t) x(s+t) d t\right| \leqq \int_{-\infty}^{\infty}|J(t)||x(s+t)| d t \\
& \leqq \beta \int_{-\infty}^{\infty}|x(s+t)| d t=\beta \int_{-\infty}^{\infty}|x(t)| d t
\end{aligned}
$$

and (4.21) follows. If it be assumed that

$$
\underset{-\infty<8<\infty}{\text { l.u.b. }}|y(s)| \leqq C \int_{-\infty}^{\infty}|x(t)| d t
$$

where

$$
C<\beta=\underset{-\infty<s<\infty}{\operatorname{m.u.b} .}|J(t)|
$$

then we can set $D=C$ in (4.51) to obtain $D=C$ in (4.55) and have a contradiction of (4.57). Therefore if $C<\beta$, then $x \varepsilon L$ exists for which (4.23) holds, and proof of Theorem 4.2 is complete.

5. Conditions for continuity of $y(s)$. The following theorem, which we give mainly for comparison with other theorems, is easily proved.

THEOREM 5.1. In order that $J(t)$ may be such that

$$
y(s)=\int_{-\infty}^{\infty} J(t) x(s+t) d t
$$

exists for all real s and is continuous whenever $x \varepsilon L$, it is necessary and sufficient that $J(t)$ be measurable and essentially bounded.

Necessity is a consequence of Theorem 2.5. If $\beta=$ m.u.b. $|J(t)|<\infty$ and $x \varepsilon L$, then the estimate

$$
\begin{aligned}
|y(s+h)-y(s)| & \leqq \int_{-\infty}^{\infty}|J(t)||x(s+h+t)-x(s+t)| d t \\
& \leqq \beta \int_{-\infty}^{\infty}|x(s+h+t)-x(s+t)| d t \\
& =\beta \int_{-\infty}^{\infty}|x(t+h)-x(t)| d t,
\end{aligned}
$$

together with the fact that $x \varepsilon L$ implies that the last member converges to 0 as $h \rightarrow 0$, shows that $y(s)$ is uniformly continuous.

It is interesting to note in connection with Theorem 5.1 and earlier theorems that the hypothesis that $y(s)$ exists and is continuous for all real $s$ whenever $x \varepsilon S$ does not imply that $J \varepsilon B$. To prove this, let $J(t)$ be a function in $L$ which is not essentially bounded and which vanishes outside some finite in- 
terval $a \leqq t \leqq b$, say $J(t)=t^{-1 / 2}$ over $0<t<1$ and $J(t)=0$ otherwise. Let $x \varepsilon S$. Then for each fixed real $s_{0}$

$$
y\left(s_{0}\right)=\int_{-\infty}^{\infty} J(t) x\left(s_{0}+t\right) d t
$$

exists since $x\left(s_{0}+t\right)$ is measurable and bounded over the finite interval $a \leqq t \leqq b$ outside of which $J(t)$ vanishes. If $K$ is chosen such that $\left|x\left(s_{0}+t\right)\right|<K$ over $a-1 \leqq t \leqq b+1$, then when $|h|<1$

$$
\begin{aligned}
\left|y\left(s_{0}+h\right)-y\left(s_{0}\right)\right| & \leqq \int_{-\infty}^{\infty}|J(t-h)-J(t)|\left|x\left(s_{0}+t\right)\right| d t \\
& \leqq K \int_{-\infty}^{\infty}|J(t-h)-J(t)| d t
\end{aligned}
$$

and, since the last integral converges to 0 with $h, y(s)$ is continuous at $s_{0}$. Thus Theorem 5.1 will fail if the phrase "whenever $x \varepsilon L$ " is replaced by the phrase "whenever $x \varepsilon S . "$

6. Some examples. Theorem 2.1 differs from Theorems 3.1 and 4.1 in that the hypothesis of Theorem 2.1 involves the special class $S_{U}$ of unit step functions while the hypotheses of Theorems 3.1 and 4.1 involve the larger class $S$. We are going to show that Theorems 3.1 and 4.1 will fail if $S$ is replaced by $S_{U}$ in their statements. For the case of Theorem 4.1, we observe that if $x \varepsilon S_{U}$ then $x \varepsilon B$ and hence that if $J \varepsilon L$ then $y \varepsilon B$; therefore the hypothesis that $y \varepsilon B$ whenever $x \varepsilon S_{U}$ does not imply that $J \varepsilon B$. For the case of Theorem 3.1, let $J(t)=e^{2 \pi i t}$. If $x \varepsilon S_{U}$, then

$$
y(s)=\int_{-\infty}^{\infty} e^{2 \pi i t} x(s+t) d t
$$

exists for each $s$ since $x(s+t) \varepsilon L$ and $e^{i t}$ is measurable and bounded; and the fact that $x(s+t)$ is constant over unit intervals, together with the fact that the integral of $e^{2 \pi i t}$ over each unit interval is 0 , implies that $y(s)=0$ and hence $y \varepsilon L$. Since $J \varepsilon L$ fails, the hypothesis that $y \varepsilon L$ whenever $x \varepsilon S_{U}$ does not imply that $J \varepsilon L$.

We show also that none of Theorems $2.1,3.1$ and 4.1 will hold if the hypotheses are relaxed to require that $y(s)$ have the stated property only when $x(t)$ is an ordinary step function. By an ordinary step function, we mean a finite linear combination of simple step functions; a simple step function being a function $\xi(t)$ such that $\xi(t)=1$ for all $t$ in the interior of some finite interval $I$ and $\xi(t)=0$ for all $t$ outside the closure of $I$. Except for the inconsequential fact that we do not require ordinary step functions to have right-hand continuity at end points of intervals, an ordinary step function may be described as a function in $S$ which vanishes outside some finite interval; hence each 
ordinary step function is equal, for all except a finite set of values of $s$, to a function in $S$.

For the case of Theorems 2.1 and 4.1 , let

$$
J_{1}(t)=t e^{i t 2} .
$$

If $x(t)=1$ when $a<t<b$ and $x(t)=0$ when $t<a$ and when $t>b$, and $y_{1}(s)$ is the $J_{1}$ transform of $x(t)$, then

$$
y_{1}(s)=\int_{a-s}^{b-s} t e^{i t 2} d t=\left[e^{i(b-s) 2}-e^{i(a-s) 2}\right] / 2 i
$$

so that $y_{1}(s)$ exists for all $s$ and is continuous over $-\infty<s<\infty$, and

$$
\left|y_{1}(s)\right| \leqq 1, \quad-\infty<s<\infty .
$$

It follows easily that the $J_{1}$ transform of each ordinary step function exists for all $s$ and is bounded and continuous. But $J_{1}$ is not essentially bounded, and the condition

$$
\underset{-\infty<u<\infty}{\operatorname{l.u.b}} \int_{u}^{u+A}\left|J_{1}(t)\right| d t<\infty
$$

fails for each $A>0$. Thus the hypothesis that $y(s)$ exists and is bounded and continuous over $-\infty<s<\infty$ whenever $x(t)$ is an ordinary step function implies neither the conclusion of Theorem 4.1 nor the conclusion of Theorem 2.1.

For the case of Theorem 3.1, let

$$
J_{2}(t)=e^{i t^{n}}
$$

where $n$ is a fixed real number greater than 2. If $x(t)=1$ when $a<t<b$ and $x(t)=0$ when $t<a$ and when $t>b$, and if $y_{2}(s)$ is the $J_{2}$ transform of $x(t)$, then we have

$$
y_{2}(s)=\int_{-\infty}^{\infty} J_{2}(t) x(s+t) d t=\int_{a-s}^{b-s} e^{i t n} d t
$$

Integration by parts gives, when $|s|$ is so great that the interval $a-s \leqq t \leqq b-s$ does not contain the origin,

$$
\left.y_{2}(s)=\frac{t^{1-n}}{i n} e^{i t n}\right]_{a-s}^{b-s}-\frac{1-n}{i n} \int_{a-s}^{b-s} t^{-n} e^{i t n} d t .
$$

Hence, for such values of $s$,

$$
\begin{aligned}
\left|y_{2}(s)\right| \leqq & n^{-1}\left[|b-s|^{1-n}+|a-s|^{1-n}\right] \\
& +(b-a)\left[|b-s|^{-n}+|a-s|^{-n}\right]
\end{aligned}
$$

so that 


$$
\limsup _{|s| \rightarrow \infty}|s|^{n-1}\left|y_{2}(s)\right| \leqq 2 / n \text {. }
$$

Since $y_{2}(s)$ is continuous, $n>2$, and (6.25) holds, we have $y_{2} \varepsilon L$. Thus the $J_{2}$ transform of each simple step function is bounded, continuous, and in $L$; and it follows that the $J_{2}$ transform of each ordinary step function also has these properties. But $J_{2} \varepsilon L$ fails. This shows that the hypothesis that $y$ is bounded, continuous, and in $L$ whenever $x$ is an ordinary step function does not imply that $J \varepsilon L$.

In case $n=2$, the transformation determined by the kernel (6.21) becomes

$$
y(s)=\int_{-\infty}^{\infty} e^{i t 2} x(s+t) d t=\int_{-\infty}^{\infty} e^{i(t-8) 2} x(t) d t
$$

and this can be written in the form

$$
\eta(s)=\int_{-\infty}^{\infty} e^{-2 i s t \xi(t) d t}
$$

where

$$
\eta(s)=y(s) e^{-i s^{2}}, \quad \xi(t)=x(t) e^{i t 2} .
$$

The function $\eta(s)$ of (6.27) differs in only a simple way from the Fourier transform of $\xi(t)$. If $\xi(t)$ is a simple step function, it is easy to compute $\eta(s)$ and to show that $\eta(s)$ is not in class $L$.

7. The class $K$ of measurable functions satisfying (2.12). The classes $L$ and $B$, and the linear vector metric complete spaces associated with them, are well known. (See, for example, the book of Banach previously cited.) In Theorem 2.1 we were led to the class $K$ of measurable functions, a member of which we now denote by $x(t)$, such that

$$
\underset{-\infty<u<\infty}{\text { l.u.b. }} \int_{u}^{u+A}|x(t)| d t<\infty
$$

for each $A>0$. The class $K$ contains all elements of $L$ and all elements of $B$. It is easy to show that the class $K$ is linear, that is, if $x_{1}, x_{2} \varepsilon K$ and $c_{1}, c_{2}$ are constants, then $c_{1} x_{1}+c_{2} x_{2} \varepsilon K$. In terms of a fixed $A>0$ and a number $\phi(A)>0$ let the norm of each $x \in K$ be defined by

$$
\|x\|=\|x\|_{A}=\underset{-\infty<u<\infty}{\text { l.u.b. } \phi(A) \int_{u}^{u+A}|x(t)| d t .}
$$

Dependence of $\|x\|$ on $A$ is illustrated by the fact that if $x_{0}(t)=|t|^{-1 / 2}$, then $x_{0} \varepsilon K$ and

$$
\left\|x_{0}\right\|=\phi(A) \int_{-A / 2}^{A / 2}\left|x_{0}(t)\right| d t=(8 A)^{1 / 2} \phi(A) .
$$


There seems to be no compelling reason why one choice of $A$ and $\phi(A)$ should be preferred over another. If $x \varepsilon L$ and $\phi(A)=1$, then $\|x\|_{A}$ converges to $\int_{-\infty}^{\infty}|x(t)| d t$ as $A \rightarrow \infty$; if $x \in B$ and $\phi(A)=1 / A$, then $\|x\|_{A}$ converges to m.u.b. $|x(t)|$ as $A \rightarrow 0$.

Assuming now that $A>0$ and $\phi(A)>0$ are fixed, it is easy to see that the class $K$ becomes a linear vector metric space when the distance between two elements $x_{1}$ and $x_{2}$ of $K$ is defined by $\left\|x_{2}-x_{1}\right\|$. We conclude by showing that this space is complete. Let $x_{1}, x_{2}, \cdots$ be a Cauchy sequence in $K$ so that $\left\|x_{m}-x_{n}\right\| \rightarrow 0$ as $m, n \rightarrow \infty$. Then as $m, n \rightarrow \infty$

$$
I_{m, n, r} \equiv \int_{r A}^{(r+1) A}\left|x_{m}(t)-x_{n}(t)\right| d t
$$

converges to 0 uniformly in $r$. Since space $L$ is complete, there is for each integer $r=0, \pm 1, \pm 2, \cdots$ a function $\xi_{r}(t)$ defined over $r A \leqq t<(r+1) A$ such that

$$
\lim _{n \rightarrow \infty} \int_{r A}^{(r+1) A}\left|\xi_{r}(t)-x_{n}(t)\right| d t=0 .
$$

If we let $\xi(t)$ be the function defined over $-\infty<t<\infty$ which agrees with $\xi_{r}(t)$ in the interval $r A \leqq t<(r+1) A$, then for each real $r$

$$
I_{n, r} \equiv \int_{r A}^{(r+1) A}\left|\xi(t)-x_{n}(t)\right| d t
$$

converges to 0 as $n \rightarrow \infty$. The inequality

$$
\left|I_{m, r}-I_{n, r}\right| \leqq I_{m, n, r},
$$

together with the fact that the right member converges to 0 uniformly in $r$ as $m, n \rightarrow \infty$ implies that the left member converges to 0 uniformly in $r$ as $m, n \rightarrow \infty$ and hence that $I_{n, r}$ converges uniformly in $r$ as $n \rightarrow \infty$. But $I_{n, r}$ converges to 0 as $n \rightarrow \infty$. Hence $I_{n, r}$ converges uniformly to 0 as $n \rightarrow \infty$, that is,

$$
\lim _{n \rightarrow \infty} \underset{-\infty<r<\infty}{\text { l.u.b. }} \int_{r A}^{(r+1) A}\left|\xi(t)-x_{n}(t)\right| d t=0,
$$

or

$$
\lim _{n \rightarrow \infty} \underset{-\infty<u<\infty}{\text { l.u.b. }} \int_{u}^{u+A}\left|\xi(t)-x_{n}(t)\right| d t=0 .
$$

This implies that $\xi \varepsilon K$, and on multiplying by the constant $\phi(A)$ we obtain $\lim \left\|\xi-x_{n}\right\|=0$. Thus each Cauchy sequence $x_{n}$ in $K$ has a limit in $K$, and completeness of the space $K$ is established.

Cornell University, ITHACA, N. Y. 